\title{
PAPER
}

\section{Green-Naghdi dynamics of surface wind waves in finite depth}

To cite this article: M A Manna et al 2018 Fluid Dyn. Res. $\mathbf{5 0} 025514$

View the article online for updates and enhancements. 


\title{
Green-Naghdi dynamics of surface wind waves in finite depth
}

\author{
M A Manna ${ }^{1}$, A Latifi $^{2}$ and R A Kraenkel ${ }^{3}$ \\ ${ }^{1}$ Université de Montpellier (UM) and Conseil National de la Recherche Scientifique \\ (CNRS), Laboratoire Charles Coulomb UMR 5221, F-34095, Montpellier, France \\ ${ }^{2}$ Department of Physics, Faculty of Sciences, Qom University of Technology, \\ Qom, Iran \\ ${ }^{3}$ Instituto de Física Téorica, UNESP-Universidade Estadual Paulista, Rua Dr. Bento \\ Teobaldo Ferraz 27, Bloco II, 01140-070, São Paulo, Brazil \\ E-mail: latifi@qut.ac.ir
}

Received 11 August 2017, revised 30 December 2017

Accepted for publication 12 January 2018

Published 8 February 2018

Communicated by Peter Haynes

\begin{abstract}
The Miles' quasi laminar theory of waves generation by wind in finite depth $h$ is presented. In this context, the fully nonlinear Green-Naghdi model equation is derived for the first time. This model equation is obtained by the non perturbative Green-Naghdi approach, coupling a nonlinear evolution of water waves with the atmospheric dynamics which works as in the classic Miles' theory. A depthdependent and wind-dependent wave growth $\gamma$ is drawn from the dispersion relation of the coupled Green-Naghdi model with the atmospheric dynamics. Different values of the dimensionless water depth parameter $\delta=g h / U_{1}$, with $g$ the gravity and $U_{1}$ a characteristic wind velocity, produce two families of growth rate $\gamma$ in function of the dimensionless theoretical wave-age $c_{0}$ : a family of $\gamma$ with $h$ constant and $U_{1}$ variable and another family of $\gamma$ with $U_{1}$ constant and $h$ variable. The allowed minimum and maximum values of $\gamma$ in this model are exhibited.
\end{abstract}

Keywords: surface waves, wind-waves, Miles' mechanism, nonlinear GreenNagdhi model, waves in finite depth

\section{Introduction}

The problem of wind-generation of surface waves has as starting point the Euler (or the Navier-Stokes) equations governing both the airflow and the surface wave dynamics. Winds generate surface wind waves and this, in its turn, modifies the airflow due to the loss of energy and momentum. In this way the atmosphere depends on the state of waves. The physical 
mechanism behind is the anti-dissipation. Energy and momentum flow continuously from the air to the surface wave. Consequently the wave amplitude grows exponentially in time more or less quickly according to the value of the growth rate which depends on the wind speed and the water depth.

It is a wonderful issue and until now several approximations and assumptions have been done to understand and describe this problem. The pioneer theoretical works to describe surface wind-waves growth began with the works of Jeffreys (1925, 1926) and Miles (1957, 1997).

In the usual Miles' theory, the water is assumed deep, the viscosity is disregarded, the equations of motions are linearized and the model has two spatial dimensions. The air domain is also considered inviscid, the equations are linearized around a prescribed mean wind velocity and air flow turbulence is only used to set up the mean wind profile.

In finite depth, the pioneer experiments and numerical studies were conduced by Thijsse (1949), Ijima and Tang (2011) and in particular, the experiments in Lake George, described by Young and Verhagen (1996a). They provide one of the first systematic attempts to understand the physics of wave-wind generation in finite depth water. The results of the field experiments in fetch limited growth have been presented in Young and Verhagen (1996a, 1996b).

In Montalvo et al (2013a) the linear Miles' mechanism of wind-wave generation was extended to finite depth. In that work, the experimental results explained only via empirical formula of Young and Verhagen (1996a, 1996b) have been derived from theoretical expressions. The wave growth rate has been determined in Montalvo et al (2013) as a function of the wave age and the wind velocities, which among the linear Miles' and Jeffreys' mechanisms would prevail. The first attempt to include the simultaneous competing effects of weakly nonlinearity, dispersion and anti-dissipation due to the wind action in finite depth was done in Manna et al (2014).

In the present paper, our aim is to derive a fully nonlinear model equation of surface wind-wave generation. Hence we are going to describe the air/water interaction from a quasi laminar point of view, in which the water obeys the fully nonlinear Green-Naghdi model and the air flow is kept, as in Miles' theory, linear and obeying the linear Euler equation of motion.

This paper is organized as follows. In section 2 the nonlinear problem of surface waves in water of finite depth is presented. In section 3 the Green-Naghdi hypothesis is introduced and a Green-Naghdi model coupled with the air dynamics is derived. Section 4 concerns the linear air domain and the associated Miles' mechanisms of surface wind waves generation. At the end of this section the Green-Naghdi model equations under the wind action via the Miles' mechanisms is exhibited for the first time. appropriate dimensionless variables and scalings in order to compute the wave growth rate are introduced in section 5. Finally section 6 draws perspectives and conclusions.

\section{The water domain}

In the water domain we consider the Euler equations for finite depth. The horizontal and vertical velocities of the fluid are $u(x, z, t)$, and $w(x, z, t)$. The continuity equation and the equations of motion read

$$
\begin{aligned}
& u_{x}+w_{z}=0, \\
& \rho_{w}\left(u_{t}+u u_{x}+w u_{z}\right)=-P_{x},
\end{aligned}
$$




$$
\rho_{w}\left(w_{t}+u w_{x}+w w_{z}\right)=-P_{z}-g \rho_{w},
$$

where $P(x, z, t)$ is the pressure, $g$ the gravitational acceleration, $\rho_{w}$ is the water density and subscripts in $u, w$ and $P$ denote partial derivatives. The boundary conditions at $z=-h$ and at $z=\eta(x, t)$ are

$$
\begin{aligned}
& w=0 ; \quad z=-h, \\
& \eta_{t}+u \eta_{x}-w=0 ; \quad z=\eta, \\
& P=P_{a} ; \quad z=\eta,
\end{aligned}
$$

where $P_{a}(x, z, t)$ is the air pressure. Thus, equation (6) is the continuity of the pressure across the air/water interface. As these are vital assumptions for the growth mechanism, let us give a more pleasant expression to these equations. So, let us introduce a reduced pressure defined by

$$
\mathbf{P}(x, z, t)=P(x, z, t)+\rho_{w} g z-P_{0},
$$

where $P_{0}$ is the atmospheric pressure. In terms of (7) equations (1)-(3) read

$$
\begin{aligned}
& u_{x}+w_{z}=0, \\
& \rho_{w}\left(u_{t}+u u_{x}+w u_{z}\right)=-\mathbf{P}_{x}, \\
& \rho_{w}\left(w_{t}+u w_{x}+w w_{z}\right)=-\mathbf{P}_{z},
\end{aligned}
$$

and (4)-(6)

$$
\begin{aligned}
& w=0 ; \quad z=-h, \\
& \eta_{t}+u \eta_{x}-w=0 ; \quad z=\eta, \\
& \mathbf{P}-\rho_{w} g \eta+P_{0}=P_{a} ; \quad z=\eta .
\end{aligned}
$$

\section{Coupling the fully nonlinear Green-Naghdi model with the atmospheric dynamics}

Shallow water model equations are usually derived by performing an asymptotic analysis directly from equations (8)-(13). Our approach is somehow different since, instead of studying the entire problem via a perturbation theory, we are going to consider the nonlinear evolution of a given Ansatz for the velocity field. We assume indeed that the horizontal velocity $u(x, z, t)$ is independent of $z$, that is

$$
u=u(x, t) \text {. }
$$

This ab initio given velocity profile is known as the columnar flow hypothesis. It was introduced long ago by Su (1969), Serre (1953) and Green and Naghdi (1974, 1976). From (8), (14) and (12) we have

$$
w(x, z, t)=-(z+h) u_{x}(x, t),
$$

so (9) and (10) read

$$
\rho_{w}\left(u_{t}+u u_{x}\right)=-\mathbf{P}_{x},
$$




$$
-\rho_{w}(z+h)\left(u_{x t}+u u_{x x}-u_{x}^{2}\right)=-\mathbf{P}_{z} .
$$

Integrating (17) and using (13) we obtain the pressure $\mathbf{P}(x, z, t)$

$$
\begin{aligned}
\mathbf{P}(x, z, t)= & \frac{1}{2} \rho_{w}\left[(z+h)^{2}-(\eta+h)^{2}\right]\left(u_{x t}+u u_{x x}-u_{x}^{2}\right) \\
& +P_{a}(x, \eta, t)+\rho_{w} g \eta-P_{0} .
\end{aligned}
$$

Substituting (18) in (16) and integrating in $z$ for $-h \leqslant z \leqslant \eta$ we have

$$
\begin{aligned}
u_{t}+u u_{x}+g \eta_{x}= & -\frac{1}{\rho_{w}}\left[P_{a}(x, \eta, t]_{x}\right. \\
& +\frac{1}{3(\eta+h)}\left\{(\eta+h)^{3}\left(u_{x t}+u u_{x x}-u_{x}^{2}\right)\right\}_{x},
\end{aligned}
$$

and (12) read

$$
\eta_{t}+[u(\eta+h)]_{x}=0
$$

If $P_{a}=P_{0}$ equations (19) and (20) are reduced to the usual Green-Naghdi equations (Green and Naghdi 1974, 1976). But this in not our case and we must determine $P_{a}(x, z, t)$ which depends on the atmospheric dynamics. So the system (19) and (20) couples the fully nonlinear Green-Naghdi model equations with the atmospheric dynamics.

\section{The Miles' mechanism in the air domain}

Let us consider the linearized (inviscid) governing equation of a steady air flow, with a prescribed mean horizontal velocity $U(z)$ depending on the vertical coordinate $z$. We are going to study perturbations to the mean flow $U(z): u_{a}(x, z, t), w_{a}(x, z, t)$ and $P_{a}(x, z, t)$ (subscript $a$ stands for air). So with $\mathbf{P}_{a}(x, z, t)=P_{a}(x, z, t)+\rho_{a} g z-P_{0}, \rho_{a}$ the air density, and $U^{\prime}=\mathrm{d} U$ $(z) / \mathrm{d} z$ we have the following equations

$$
\begin{aligned}
& u_{a, x}+w_{a, z}=0, \\
& \rho_{a}\left[u_{a, t}+U(z) u_{a, x}+U^{\prime}(z) w_{a}\right]=-\mathbf{P}_{a, x}, \\
& \rho_{a}\left[w_{a, t}+U(z) w_{a, x}\right]=-\mathbf{P}_{a, z},
\end{aligned}
$$

which must be completed by some appropriate boundary conditions. The first one is the kinematic boundary condition for air, evaluated at the aerodynamic sea surface roughness $z_{0}$ placed just above the interface. It reads

$$
\eta_{t}+U\left(z_{0}\right) \eta_{x}=w_{a}\left(z_{0}\right)
$$

Through this paper, $z_{0}$ will be regarded as a constant, independent from the sea state. This is a widely used approximation, first proposed by Charnock (1955). For the datasets used later on, the wind speed ranges are such that the roughness may be seen as a constant (Fairall et al 1996).

We choose $U(z)$ to be the following wind profile

$$
U(z)=U_{1} \ln \left(z / z_{0}\right), \quad U_{1}=\frac{u_{*}}{\kappa}, \quad \kappa \approx 0.41,
$$

where $u_{*}$ is the friction velocity and $\kappa$ the Von Kármán constant. This logarithmic wind profile is commonly used to describe the vertical distribution of the horizontal mean wind speed within the lowest portion of the air-side of the marine boundary layer 
(Garratt et al 1996, Tennekes 1972). With (25) the equation (24) reduces to

$$
\eta_{t}=w_{a}\left(z_{0}\right)
$$

This equation describes the influence of the surface perturbation dynamics $\left(\eta_{t}\right)$ on the atmosphere (through the perturbed wind speed $w_{a}\left(z_{0}\right)$ ). Consequently the air feels the water. So, the atmosphere dynamics (beyond, the initial logarithmic profile $U(z)$ ) is determined by the surface wave dynamics.

Now we assume $\mathbf{P}_{a}=\mathcal{P}_{a}(z) \exp (\mathrm{i} \theta), \quad u_{a}=\mathcal{U}_{a}(z) \exp (\mathrm{i} \theta), \quad w_{a}=\mathcal{W}_{a}(z) \exp (\mathrm{i} \theta) \quad$ with $\theta=x-c t, c$ being the phase speed and we add the following boundary conditions on $\mathcal{W}_{a}$ and $\mathcal{P}_{a}$,

$$
\begin{aligned}
& \lim _{z \rightarrow+\infty}\left(\mathcal{W}_{a}^{\prime}+k \mathcal{W}_{a}\right)=0, \\
& \lim _{z \rightarrow z_{0}} \mathcal{W}_{a}=W_{0}, \\
& \lim _{z \rightarrow+\infty} \mathcal{P}_{a}=0
\end{aligned}
$$

that is, the disturbance plus its $z$-derivative vanish at infinity, and the vertical component of the wind speed is enforced by the wave movement at the sea surface. Then, using equations (21)-(23) and (29) we obtain

$$
\begin{aligned}
& w_{a}(x, z, t)=\mathcal{W}_{a} \exp (\mathrm{i} \theta), \\
& u_{a}(x, z, t)=\frac{\mathrm{i}}{k} \mathcal{W}_{a, z} \exp (\mathrm{i} \theta), \\
& \mathbf{P}_{a}(x, z, t)=\mathrm{i} k \rho_{a} \exp (\mathrm{i} \theta) \int_{z}^{\infty}[U-c] \mathcal{W}_{a} \mathrm{~d} z^{\prime} .
\end{aligned}
$$

Removing the pressure from the Euler equations, we find the well-known Rayleigh equation $\forall z \backslash z_{0}<z<+\infty$ (Rayleigh 1880, Conte and Miles 1959)

$$
(U-c)\left(\mathcal{W}_{a}^{\prime \prime}-k^{2} \mathcal{W}_{a}\right)-U^{\prime \prime} \mathcal{W}_{a}=0,
$$

which is singular at $z_{c}=z_{0} \mathrm{e}^{c \kappa / u_{*}}>z_{0}>0$, where $U\left(z_{c}\right)=c$. We calculate $P_{a}(x, \eta, t)$

$$
P_{a}(x, \eta, t)=P_{0}-\rho_{a} g \eta+c k^{2} \rho_{a} \frac{\eta}{W_{0}} \int_{z_{0}}^{\infty}[U-c] \mathcal{W}_{a} \mathrm{~d} z
$$

where the lower integration bound is taken at the roughness height $z_{0}$ instead of $z=\eta$ since we are studying the linear problem and we have used equation (26) to eliminate the term $\mathrm{i} k \rho_{a} \exp (\mathrm{i} \theta)$. Now in terms of the integrals $I_{1}$ and $I_{2}$ defined as follow

$$
I_{1}=\int_{z_{0}}^{\infty} U \mathcal{W}_{a} \mathrm{~d} z, \quad I_{2}=\int_{z_{0}}^{\infty} \mathcal{W}_{a} \mathrm{~d} z
$$

Equation (34) reads

$$
P_{a}(x, \eta, t)=P_{0}-\left[g-\frac{c k^{2}}{W_{0}}\left(I_{1}-c I_{2}\right)\right] \rho_{a} \eta .
$$

In equations (30)-(31) and (36) neither $\mathcal{W}_{a}$ nor $c$ are known. In order to find $c$ we use the linear system associated with (19) and (20) and equation (36)

$$
u_{t}+g \eta_{x}(1-s)+s k^{2} c \frac{\eta_{x}}{W_{0}}\left(I_{1}-c I_{2}\right)+\frac{h^{2}}{3} u_{x x t}=0,
$$




$$
\eta_{t}+h u_{x}=0 .
$$

Now, writing $u=u_{0} \mathrm{e}^{\mathrm{i} \theta}$ and $\eta=\eta_{0} \mathrm{e}^{\mathrm{i} \theta}$ and substituting them in (37), (38) yields to a linear system of equations for $u_{0}$ and $\eta_{0}$. The determinant of this system gives the following equation

$$
c^{2}\left\{1+\frac{k^{2} h^{2}}{3}+s \frac{k^{2} h}{W_{0}} I_{2}\right\}-s \frac{c k^{2} h-1}{W_{0}}-g h(1-s)=0 .
$$

The parameter $s=\rho_{a} / \rho_{w}$ is small $\left(\sim 10^{-3}\right)$ and allow us to expand $c$

$$
c=\mathbb{A}+\mathbb{B} s+O\left(s^{2}\right) .
$$

Substituting (40) in (39), yields at order $s^{0}$

$$
\mathbb{A}=c_{0}=\sqrt{\frac{g h}{1+\frac{k^{2} h^{2}}{3}}} .
$$

and at order $s^{1}$

$$
\mathbb{B}=-\frac{c_{0}}{2}+\frac{c_{0} k^{2}}{2 g W_{0}}\left(c_{0} I_{1}-c_{0}^{2} I_{2}\right) .
$$

The relation (39) reads then

$$
c=c_{0}\left(1-\frac{s}{2}\right)+s \frac{c_{0} k^{2}}{2 g W_{0}}\left(c_{0} I_{1}-c_{0}^{2} I_{2}\right)+O\left(s^{2}\right) .
$$

If $s=0$ we obtain the classical dispersion relation of the Green-Naghdi equations (Green and Naghdi 1976)

$$
\omega_{0}=k c_{0}=k \sqrt{\frac{g h}{1+\frac{k^{2} h^{2}}{3}}} .
$$

Now substituting (43) in (33) and neglecting the products of $s$ with perturbative quantities (and their derivatives) the Rayleigh equation can be solved. This allows us to compute $I_{1}$ and $I_{2}$.

Due to the complexe form of $w_{a}(x, z, t)(30)$, the term $I_{1}-c_{0} I_{2}$ is complex (see Beji and Nadaoka 2004), so we can write

$c=c_{0}+s\left\{-\frac{c_{0}}{2}+\frac{c_{0}^{2} k^{2}}{2 g W_{0}}\left[\operatorname{Re}\left(I_{1}-c_{0} I_{2}\right)\right]\right\}+s \frac{c_{0}^{2} k^{2}}{2 g W_{0}} \mathrm{i}\left[\mathbf{I m}\left(I_{1}-c_{0} I_{2}\right)\right]$.

The linear expression of $P_{a}(x, \eta, t) / \rho_{w}$ can be written at order $s$ as

$P_{a} / \rho_{w}=P_{0} / \rho_{w}-\left[g-\frac{c_{0} k^{2}}{W_{0}} \mathbf{R e}\left(I_{1}-c_{0} I_{2}\right)\right] s \eta+\frac{c_{0} k}{W_{0}} \mathbf{I m}\left(I_{1}-c_{0} I_{2}\right) s \eta_{x}$.

Equations (46) and (19) yield the Green-Naghdi model equations under the linear windaction. They read

$$
\begin{aligned}
u_{t}+u u_{x}+g \eta_{x}= & {\left[g-\frac{c_{0} k^{2}}{W_{0}} \operatorname{Re}\left(I_{1}-c_{0} I_{2}\right)\right] s \eta_{x}-\frac{s c_{0} k}{W_{0}} \operatorname{Im}\left(I_{1}-c_{0} I_{2}\right) \eta_{x x} } \\
& +\frac{1}{3(\eta+h)}\left[(\eta+h)^{3}\left(u_{x t}+u u_{x x}-u_{x}^{2}\right)\right]_{x},
\end{aligned}
$$




$$
\eta_{t}+[u(\eta+h)]_{x}=0 .
$$

The first term at the right-hand side of (47) changes the linear phase velocity of the system. The second one is an anti-dissipative term. This term transports (continuously) energy from the wind to the wave. The last terms at the right-hand side of (48) are the well known nonlinear and dispersive terms of the usual Green-Naghdi equations (Green and Naghdi 1974, 1976).

\section{The finite depth parameters}

In this section we introduce suitable non-dimensional variables. The problem is nondimensionalized using the undisturbed depth of the water $h$, a typical wavenumber $k$ and the acceleration due to gravity $g$. It follows that the appropriate non-dimensionalization for the horizontal velocities (in the water and in the air) is the typical wind velocity $U_{1}$ (defined in (25)), the appropriate non-dimensionalization for the vertical wind velocity perturbation is $W_{0}$ and the appropriate time scale is $U_{1} / g$. So we have

$$
\begin{aligned}
& x=h \hat{x}, \quad z=h \hat{z}, \quad \eta=h \hat{\eta}, \quad t=\frac{U_{1}}{g} \hat{t}, \quad U=U_{1} \hat{U}, \\
& u=U_{1} \hat{u}, \quad c_{0}=U_{1} \hat{c_{0}}, \quad \mathcal{W}_{a}=W_{0} \hat{\mathcal{W}}_{a},
\end{aligned}
$$

where hats mean dimensionless variables. With theses definitions the Green-Naghdi equations under the wind-action are transformed in a dimensionless system which reads (dropping the hats)

$$
\begin{aligned}
\delta u_{t}+u u_{x}+\delta \eta_{x}= & {\left[\delta-\epsilon^{2} c_{0} \mathbf{R e}\left(I_{1}-c_{0} I_{2}\right)\right] s \eta_{x}-\epsilon c_{0} \mathbf{I m}\left(I_{1}-c_{0} I_{2}\right) s \eta_{x x} } \\
& +\frac{1}{3(\eta+1)}\left[(\eta+1)^{3}\left(\delta u_{x t}+u u_{x x}-u_{x}^{2}\right)\right]_{x} \\
\delta \eta_{t}+ & {[u(\eta+1)]_{x}=0 }
\end{aligned}
$$

where the dimensionless parameters $\epsilon, \delta$ and $c_{0}$, are defined as

$$
\epsilon=k h, \quad \delta=\frac{g h}{U_{1}^{2}}, \quad c_{0}=\frac{\delta^{1 / 2}}{\left(1+\epsilon^{2} / 3\right)^{1 / 2}} .
$$

Obviously $\epsilon$ is the parameter measuring dispersion. The parameter $\delta$ is the ratio of the square of the dispersionless phase velocity of the system $\sqrt{g h}$ and the square of the wind velocity $U_{1}$. $c_{0}$ is the ratio of the phase velocity of the usual Green-Naghdi equations and $U_{1}$. A given value of $\sqrt{\delta}$ characterizes the relative value between the extreme linear phase velocity and the wind.

The allowed values of $\epsilon$ and $c_{0}$ are

$$
0<\epsilon<1, \quad \sqrt{\frac{3 \delta}{4}}<c_{0}<\sqrt{\delta} .
$$

Parameters $\epsilon$ and $\delta$ are two independent dimensionless parameters.

Imagine a constant wind in direction and magnitude blowing perpendicular to an ideal straight lake's coastline. In the region where the wind meets the water, short surfaces waves are generated. As the waves progress, theirs phase velocities increase such that at intermediate distances the wavelengths are largest. Hence at largest distance from the shoreline, the phase velocities are constant and $\sim \sqrt{\delta}$. A suitable parameter able to describe this pattern is $c_{0}$. The phase velocity grows running from a minimum value $\sim \sqrt{\frac{3 \delta}{4}}$ (for maximum dispersion with 
$\epsilon \sim 1$ ) to a maximum value $\sim \sqrt{\delta}$ (for minimum dispersion $\epsilon \sim 0$ ). Consequently, $c_{0}$ is a kind of theoretical wave-age.

The term $\left(\delta-\epsilon^{2} c_{0} \operatorname{Re}\left(I_{1}-c_{0} I_{2}\right)\right) s \eta_{x}$ can be disregarded because it contributes only to the linear phase velocity without introducing any changes on the growth rate of the waves. With this approximation, the dimensionless phase velocity of the system (50), (51) is given by

$$
c=c_{0}+\mathrm{i} \frac{\epsilon^{2} c_{0}^{2} s}{2 \delta} \operatorname{Im}\left(I_{1}-c_{0} I_{2}\right) \text {. }
$$

Now $c_{0}$ together with the linear progressive dispersionless wave solution $\eta \sim \exp (\epsilon x-(\epsilon / \delta) t)$ yield

$$
\eta \sim \exp \left[\mathrm{i} \epsilon\left(x-\frac{c_{0}}{\delta}\right)\right] t \times \exp \left[\frac{\epsilon^{3} c_{0}^{2} s}{2 \delta^{2}} \mathbf{I m}\left(I_{1}-c_{0} I_{2}\right)\right] t .
$$

The real exponential determines the growth rate of the the plane wave solution $\gamma$,

$$
\gamma\left(\epsilon, \delta, c_{0}\right)=\frac{\epsilon^{3} c_{0}^{2} s}{2 \delta^{2}} \mathbf{I m}\left(I_{1}-c_{0} I_{2}\right) .
$$

The existence of a finite depth $h$ transforms the uniquely defined function of wave growth rate in deep water in families of functions indexed by $\delta=g h / U_{1}^{2}$, i.e., a function for each value of $\delta$. A family of $\gamma$-functions is obtained with $h$ constant and $U_{1}$ variable and another family of $\gamma$-functions can be obtained if $U_{1}$ is constant and $h$ is variable.

There exist two extreme limits for $\gamma\left(\gamma_{\max }\right.$ and $\left.\gamma_{\min }\right)$ obtained from two extreme limits for the wave age $c_{0}\left(c_{0}^{1}\right.$ and $\left.c_{0}^{2}\right)$

$$
\begin{aligned}
& c_{0}^{1}=\sqrt{\frac{3 \delta}{4}}, \quad \rightarrow \gamma_{\max }=\frac{3 s}{8 \delta} \mathbf{I m}\left(I_{1}-\sqrt{\frac{3 \delta}{4}} I_{2}\right), \\
& c_{0}^{2}=\sqrt{\delta}, \quad \rightarrow \gamma_{\min }=0 .
\end{aligned}
$$

Expression (57) and (58) are the maximum and the minimum growth rate in this model.

\section{Perspectives and conclusions}

We have introduced a quasi laminar Miles' mechanism of wind waves generation in finite depth. We have linearized the equations of motion governing the air dynamics but the water dynamics was considered fully nonlinear and obeying to the Green-Naghdi model equation. The model is in addition weakly dispersive and the action of the wind appears by an antidissipative term. Anti-dissipation causes growth of the free water surface and we have computed the wave growth of the plane wave solutions $\gamma$ in terms of the dimensionless parameters $\epsilon, \delta$ and $c_{0}$. The extreme value $\gamma_{\max }$ is obtained for the minimum value of the wave age $c_{0}^{1}=\sqrt{3 \delta / 4}$ according to the fact that in young seas the surface waves are short with large aspect ratios, so the wind anti-dissipative action is maximal in this case. The extreme value $\gamma_{\min }=0$ is obtained for the maximum value of the wave age $c_{0}^{2}=\sqrt{\delta}$ according to the fact that in old seas the surface waves are large with small aspect ratios, so the wind antidissipative action is minimal.

It worth remarking that although the Green-Naghdi equation gives an excellent approximation of one layer fluid (Lannes 2013), it does not improve significantly the basic ill-posedness of classical two layer shallow water model (Liska et al 1995). Indeed, according to Liska et al (1995) (figure 1) for small $s=\rho_{a} / \rho_{w}$ there exists a narrow region of 
ill-posedness which corresponds to the region around the singularity of the equation (33) where the energy transfer from the air to water operates through resonance.

Some important analytical and numerical works are still to be done in upcoming studies. Namely, a numerical study of the Rayleigh equation in order to compute (explicitly) $I_{1}$ and $I_{2}$, the blow-up in finite time associated with the soliton solution of the anti-dissipative GreenNaghdi model (47) and (48) and last but not least, the comparison of our analytical and numerical results with experimental results.

\section{Acknowledgments}

This work has been supported by the Center for International Scientific Studies and Collaboration (CISSC).

\section{References}

Beji S and Nadaoka K 2004 J. Fluid Mech. 50065

Charnock H 1955 Q. J. R. Meteorol. Soc. 81639

Conte S and Miles J 1959 J. Soc. Ind. Appl. Math. 7361

Fairall C W, Grachev A A, Bedard A and Nishiyama R T 1996 NOAA Technical Memorandum ERL ETL-268

Garratt J, Hess G, Physick W and Bougeault P 1996 Bound.-Layer Meteorol. 789

Green A E and Naghdi P M 1974 Proc. R. Soc. A 337451

Green A E and Naghdi P M 1976 J. Fluid Mech. 78237

Ijima T and Tang F L W 2011 Coastal Engineering Proc. (North America)

Jeffreys H 1925 Proc. R. Soc. A 107189

Jeffreys H 1926 Proc. R. Soc. A 110241

Lannes D 2013 The Water Waves Problem: Mathematical Analysis and Asymptotics (Mathematical Surveys and Monographs) (Providence, RI: American Mathematical Society)

Liska R, Margolin L and Wendroff B 1995 Comput. Math. Appl. 29 25-37

Manna M A, Montalvo P and Kraenkel R A 2014 Phys. Rev. E 90013006

Miles J 1957 J. Fluid Mech. 3185

Miles J 1997 Appl. Mech. Rev. 50 R5

Montalvo P, Dorignac J, Manna M, Kharif C and Branger H 2013a Coast. Eng. 7749

Montalvo P, Kraenkel R, Manna M A and Kharif C 2013b Nat. Hazards Earth Syst. Sci. 132805

Rayleigh L 1880 Proc. London Math. Soc. XI 57

Serre F 1953 Houille Blanche 3374

Su C S and Gardner C S 1969 J. Math. Phys. 10536

Tennekes H 1972 J. Atmos. Sci. 30234

Thijsse J T 1949 Gen. Assem. Assoc. d'Océanographie Phys., Procés-Verbaux 480

Young I R and Verhagen L A 1996a Coastal Eng. 2947

Young I R and Verhagen L A 1996b Coastal Eng. 2979 